\title{
The AFRL-MITLL WMT16 News-Translation Task Systems
}

\author{
Jeremy Gwinnup, Timothy Anderson, \\ Grant Erdmann, Katherine Young \\ Air Force Research Laboratory \\ \{jeremy.gwinnup.1, timothy . anderson. 20, \\ grant . erdmann, katherine . young. 1. ctr\}@us . af .mil
}

\author{
Michaeel Kazi, Elizabeth Salesky, \\ Brian Thompson \\ MIT Lincoln Laboratory \\ \{michaeel.kazi, elizabeth.salesky, \\ brian.thompson\}@ll.mit.edu
}

\begin{abstract}
This paper describes the AFRL-MITLL statistical machine translation systems and the improvements that were developed during the WMT16 evaluation campaign. New techniques applied this year include Neural Machine Translation, a unique selection process for language modelling data, additional out-of-vocabulary transliteration techniques, and morphology generation.
\end{abstract}

\section{Introduction}

As part of the 2016 Conference on Machine Translation (WMT16) news-translation shared task, the MITLL and AFRL human language techology teams participated in the Russian-English and English-Russian news translation tasks. Our machine translation (MT) systems represent improvements to both our systems from IWSLT2015 (Kazi et al., 2015) and WMT15 (Gwinnup et al., 2015), the introduction of Neural Machine Translation rescoring, neural-net based recasing, unsupervised transliteration of out-of-vocabulary (OOV) words (Durrani et al., 2014), and an unique selection process for language modelling data. For the English-Russian translation task we experimented with techniques to improve morphology generation.

\section{System Description}

We submitted systems for the Russian-English and English-Russian news-domain machine translation shared tasks. For all submissions, we used the phrase-based variant of the Moses decoder (Koehn et al., 2007). As in previous years, our submitted

\footnotetext{
This work is sponsored by the Air Force Research Laboratory under Air Force contracts FA-8721-05-C-0002 and FA8650-09-D-6939-029.
}

systems used only the constrained data supplied when training.

\subsection{Data Usage}

In training our systems we drew on all the available data, filtering the new English Common Crawl monolingual data as described in $\$ 2.4$ and $\$ 3.1$. The Wikipedia Headlines corpus ${ }^{1}$ was reserved to train a neural network based transliteration system described in $\S 2.8 .1$.

\subsection{Data Preprocessing}

We processed the training data similarly to our WMT15 system (Gwinnup et al., 2015). We examined irregular behaviors in Moses's punctuation normalization script ${ }^{2}$. We ran a script that examines the source and target side of the parallel training data and removes lines that are identical in both the source and target in order to prevent the effects of wrong-language phrases "polluting" the phrase and rule tables.

\subsection{Phrase Table Generation}

We used the standard Moses method of extracting and creating phrase tables. Phrase tables were binarized using either the Compact Phrase Table (Junczys-Dowmunt, 2012) or ProbingPT (Bogoychev and Hoang, 2016) methods.

\subsection{Language Model Data Selection}

Using definitions below, we select as a language modelling set a subset $S$ from the Common Crawl set $C$ to maximize its similarity to a target set $T$, using a coverage metric $g(S, T)$. Defining $c_{i}(X)$ as the count of feature $i$ 's occurrence in corpus $X$,

$$
g(S, T)=\frac{\sum_{i \in \mathcal{I}} f\left(\min \left(c_{i}(S), c_{i}(T)\right)\right)}{\sum_{i \in \mathcal{I}} f\left(c_{i}(T)\right)+p_{i}(S, T)}
$$

\footnotetext{
${ }^{1}$ http://statmt.org/wmt15/wiki-titles.tgz

${ }^{2}$ normalize-punctuation.perl
} 
where the oversaturation penalty $p_{i}(S, T)$ is

$\max \left(0, c_{i}(S)-c_{i}(T)\right)\left[f\left(c_{i}(T)+1\right)-f\left(c_{i}(T)\right)\right]$.

We use $f(x)=\log (1+x)$ as the submodular function to weight counts, and the feature set $\mathcal{I}$ is the set of all unigrams and bigrams. The target set $T$ is made of the news test sets from 2013-2015.

The optimization problem, $\max _{S \subset C} g(S, T)$, is solved via greedy optimization, iteratively adding the segment to $S$ that provides the largest increase in $g$. The set $S$ is reviewed after each addition, removing any older segment in $S$ that decreases $g$.

The Common Crawl corpus $C$ is broken into easily-processed chunks of ten thousand segments, selecting five hundred segments from each chunk. This selection was repeated until we saw diminishing returns from adding further chunks, resulting in a language modelling subset of six million lines. These six million lines represent $0.17 \%$ of the 3.6 billion lines of data in the English portion of the Common Crawl.

\subsection{Tuning Improvements}

Improvements were made to our tuner, Drem (Erdmann and Gwinnup, 2015), since our last submission. Enforcement of minimum and maximum distance of the tuning result from prior decodes (i.e., tabu and fear constraints) is now implicitly enforced via $L_{1}$ penalty functions, making the process more robust to densely-packed decodes. Rescoring weights are now not penalized in the n-best list interpolation scheme, since they do not directly affect n-best lists. This new feature provides faster convergence of our NMT-rescored systems. Another improvement to Drem is that the metric chrF3 (Popović, 2015) is now available as a tuning objective function.

\subsection{Neural Network Recaser}

We noticed a substantial gap between uncased and cased BLEU scores on our systems. Addressing the problem in post-processing, it became apparent that recasing can only do so much on monolingual data. We therefore built a classifier that uses both the source-side and the target-side of the translations. The inputs to the classifier are:

- $t_{i}$, the word to be recased, as well as $t_{i-1}$ and $t_{i-2}$

- $s_{a(i)}$, the source word aligned to $t_{i}$, plus $s_{a(i) \pm 1}$. Alignments were taken from Moses output, and missing alignments were computed using the NNJM affiliation heuristic (Devlin et al., 2014).

- The status of the source word as lowercase, capitalized, or OTHER.

The exact classifier used could be anything; we chose a neural network because it is simple to create and robust. Our architecture is as follows:

1. Vocabulary of all words, excluding $25 \%$ of singletons

2. Input: Word vectors for these words, plus nine binary inputs $\left(s_{i-1}=l c, s_{i-1}=\right.$ $\left.U c, s_{i-1}=O T H E R, s_{i}=l c \ldots\right)$, all concatenated together into a single vector

3. Two hidden layers, default size 100

4. One softmax output, 3 output classes

The resulting recaser consistently yields $+0.2-$ 0.25 case-sensitive BLEU over a standard language model recaser.

\subsection{Inflection Generation}

English-Russian systems have the added challenge of generating morphologically rich word-forms. In addition to an English-Russian baseline, we trained two methods to generate inflected forms. First, we created a system with a separate inflection prediction component (Toutanova et al. 2008, Fraser et al. 2012). We trained an MT system from English to lemmatized Russian, using the Mystem $^{3}$ Russian morphological analyzer to lemmatize all available parallel data, and then trained a MT system from lemmatized Russian to Russian. Scoring against lemmatized references, the first step yielded 27.70 case-insensitive BLEU on newstest2016. However, while the lemru-ru system was successful with one-to-one lemmatized training data, it couldn't recover from mistakes in the MT output of the first step and the system overall did not perform as well as our baseline (17.19 cased BLEU).

We also attempted to address inflection generation during training using verb annotation, following the approach of Kirchhoff et al. (2015) for Arabic verb inflection. We use dependency parsing to identify the subject of the verb in the English sentence and then annotate the verb with the person and number of the subject. With a pronominal subject he or she, the verb is also annotated for gender.

\footnotetext{
${ }^{3}$ https://api .yandex.ru/mystem
} 
Original: Woud n't you know it ?

Annotated: Would n't you know-2p it ?

Dependency Parse:

\begin{tabular}{lllll}
\hline Index & Word & POS & Head & Relation \\
\hline 1 & Would & MD & 4 & aux \\
2 & n't & RB & 4 & neg \\
3 & you & PRP & 4 & nsubj \\
4 & know & VB & 0 & root \\
5 & it & PRP & 4 & dobj \\
6 & $?$ &. & 4 & punct \\
\hline
\end{tabular}

Figure 1: Annotation via Dependency Parse

This provides the potential for the system to match annotated English verbs to the correctly inflected Russian verbs during training. Figure 1 shows an annotated sentence and the underlying dependency parse.

We use the Stanford parser (Klein and Manning, 2003) and conversion utility to generate the dependency parses, adjusting the tokenization of the input to match the Stanford treatment of contractions. We apply annotation to verbs with subjects listed as nsubj or xsubj in the dependency parse. Person, number, and gender are derived from the subject's POS tag and from the specific lexical item for pronouns. Coordinate subjects are counted as plural.

An unannotated MT system has a good chance of associating the correct verb form with the subject if the subject and verb are adjacent and can be extracted as a phrase, while more distant pairs are less likely to be found in the phrase table, leaving the verb open to translation in the wrong inflected form. Since annotation can increase data sparsity, it is better to apply it only when necessary.

Kirchhoff et al. (2015) address the data sparsity issue by only applying their annotation-trained model when their baseline model translates the subject and verb via separate phrases. In some of our systems, we simulated the use of a backoff model by restricting our annotation to subjects and verbs that occur with a minimum separation distance.

Figure 2 shows the potential effect of specifying a minimum separation distance. In the first sentence, the subject and verb are adjacent; any separation requirement greater than zero prevents annotation of the verb. The other sentences show a greater separation, and annotation will be main-
Would n't you know-2p it?

The country was gradually recovering- $3 p-s g$.. The interests of people take-3p-pl precedence ..

Figure 2: Annotation at different separation distances.

tained if the separation requirement is less than 3 .

In order to avoid the data sparsity problem, we ultimately created a factored version of the verb annotation system. The annotations were specified as factors on the verb, with a null factor on the unannotated words, e.g. would/NONE $n$ ' t I NONE you/NONE know $2 p$ it|NONE ? INONE

In system 2 of our English-Russian systems (shown in Table 8), we used this factored input with no separation limit.

\subsubsection{Discussion}

We examined the effect of verb annotation on inflection choice using an enhanced version of the Hjerson (Popović, 2011) error analysis program, in conjunction with the Mystem Russian morphological analyzer. Factored verb annotation as described above failed to reduce the number of inflectional errors (shown in Table 1.)

\begin{tabular}{lcc}
\hline Technique & Inf. Errors & Pct. Hyp. Words \\
\hline Baseline & 5823 & $9.349 \%$ \\
Annotated & 5994 & $9.351 \%$ \\
\hline
\end{tabular}

Table 1: Hjerson performance

The verb annotation technique aims to increase the information available for the generation of verb inflections. Errors in verb inflection amount to just a small proportion of overall errors in our baseline system, so the room for improvement in translation quality is small (shown in Table 2.)

\begin{tabular}{lcc}
\hline Error Type & Instances & Pct. Hyp. Words \\
\hline Word Choice & 30031 & $48.21 \%$ \\
Reordering & 4479 & $7.19 \%$ \\
Inflection & 5823 & $9.35 \%$ \\
\hline
\end{tabular}

Table 2: Hjerson classification of Error Types in Baseline System

Only about $18 \%$ of these 5823 baseline inflectional errors involve verbs; other errors involve nouns and pronouns (about 58\%) or adjectives 
(about 24\%). Meanwhile, the use of annotated data had unintended consequences for the other elements in the sentence. While our annotations were only applied to verbs in the training data, changes in inflection were observed for nouns and pronouns as well.

We used Mystem to provide a morphological analysis of the inflectional errors. We found that similar errors were made in both the baseline system and the annotated system. Looking at the error types by part of speech, we saw that verb errors for both systems primarily involved either number or gender, as opposed to tense or person. Pronoun errors for both systems showed a tendency for oblique cases in place of nominative.

For example, both systems displayed errors in which будут (third person plural) "they will" was generated instead of the reference form, будет (third person singular) "he will". The baseline system had 8 instances of this error, while the annotated system had 10 instances. The most frequent error was the substitution of the dative/locative first person singular pronoun мне "to me" for the nominative pronoun я "I". The baseline system had 16 instances of this error, compared to 20 instances for the annotated system.

The verb-annotated system performed worse than our baseline when evaluated with the BLEU metric. We hope to gain more insight from the human ranking of the two systems.

\subsection{Transliteration}

We employed two methods to address transliteration of remaining out-of-vocabulary (OOV) words: an unsupervised statistical transliteration approach and a novel character-based neuralnetwork transliteration approach.

\subsubsection{Neural Network Transliteration}

We created a list of 54k Named Entity (NE) pairs from the Common Crawl using transliteration mining; we also derived NE pairs from the Wikipedia Headlines Corpus (Gwinnup et al., 2015). We employed these lists in building a neural network based transliterator. We trained an encoderdecoder LSTM network to produce characters in a target language given characters from a word in the source language. The network configuration was nearly the same as that in our NMT experiments, except the network was significantly smaller (hidden sizes of 100 and 200, with 1,2 , and 3 hidden layers) and had a beam of 5 . A small (5k) sub- set of the data was held out for evaluation/tuning. Since Russian nouns use case inflections, multiple Russian word forms may map to a single English spelling. For this reason, we tried rescoring with a unigram language model trained on the monolingual data to help weight the correct English spelling of words that may have been seen in the language modelling data but were not in the phrase table. The LM's unknown word probability was optimized on the validation set.

\begin{tabular}{lc}
\hline System & Exact matches \\
\hline Baseline [0 edit distance] & $23.1 \%$ \\
Single enc-dec & $34.7 \%$ \\
Ensemble (6) & $38.7 \%$ \\
Single enc-dec + LM rescore & $42.5 \%$ \\
Ensemble (6) + LM rescore & $45.8 \%$ \\
\hline
\end{tabular}

Table 3: Fraction of transliterations that match exactly, on validation set (subset of newstest2014)

We integrated this process into our SMT pipeline through different backoff phrase tables. Unknown words from the dev and test sets were transliterated via beam search (beam and stack size of 5) using the final system in Table 3 to create phrase table entries. The results are in Table 4. Gains may seem modest, however, there are not that many OOV words in newstest2015 - only 817 total unknowns, 515 of which we attempted to transliterate (ASCII entries and Capitalized words). Despite this, gains are consistent.

\begin{tabular}{lc}
\hline System & Cased BLEU \\
\hline 1. drop unknowns & 28.07 \\
2. pass-through unknowns & 27.85 \\
3. ASCII entries in backoff PT & 27.86 \\
4. 3 + cased words LM match & 28.20 \\
5. 3 + all cased Cyrillic words & 28.16 \\
\hline
\end{tabular}

Table 4: Neural Transliteration via Backoff PTs

\subsubsection{Unsupervised Statistical Transliteration}

As a contrast to our neural network transliteration approach, we also experimented with using the unsupervised statistical transliteration method (Durrani et al., 2014) included in Moses. System 2 in Table 7 and both systems in Table 8 employ this strategy as a post-decode step. 


\subsection{Neural MT}

We describe a Neural Machine Translation system we developed and our strategies to integrate this system into our machine translation framework.

\subsubsection{System}

We trained a neural encoder-decoder network (Sutskever et al., 2014; Bahdanau et al., 2014; Luong et al., 2015) using the attention model from (Vinyals et al., 2015) to perform neural machine translation (NMT). We trained the model using Adagrad (Duchi et al., 2011) and found it improved performance over the learning rate schedule proposed in (Luong et al., 2015). We also found it advantageous to use a larger source vocabulary $(200 \mathrm{k}-500 \mathrm{k}$ words worked well). Each instance of the system was comprised of two 1000-dim hidden layers, with beam and stack of 5. Our NMT results are shown in Table 5. They did not perform competitively with our SMT systems by themselves, however they were very useful in rescoring as others have noted (Auli et al., 2013).

\begin{tabular}{lc}
\hline System & Cased BLEU \\
\hline 1. Single model & 21.00 \\
2. Ensemble of 2 & 21.46 \\
\hline
\end{tabular}

Table 5: Russian-English Neural MT Systems decoding newstest 2015

\subsubsection{Reranking}

We compared two different ways of using the NMT system to augment our phrase-based system.

1. Single set of weights We augment the Moses n-best list with NMT scores for each sentence, and then tune the decode weights using Drem. We repeat this process 10 times, using the last weights to decode the test set and one-best calculation.

2. Decode + rerank weights We tune the decode weights using Drem, without the NMT scores. After 10 iterations, we merge the nbest lists together and compute NMT scores over the result. Then, we compute a second set of weights. To decode the test set, we pass the decode weights to Moses, augment the nbest list with NMT scores, and finally apply the one-best dot product using the second set of weights.

\begin{tabular}{lc}
\hline Features & Cased BLEU tst15 \\
\hline $\mathrm{pb}+$ BigLM & 27.09 \\
$+\mathrm{nmt}$ & 27.92 \\
$+\mathrm{cc} \mathrm{LM}$ data & 28.07 \\
+ translit & 28.20 \\
\hline
\end{tabular}

Table 6: Score breakdown for en-ru submission system 1, average of 6 runs on newstest 2015 .

The first process produced scores of 27.22, and the second 27.92 (mteval, case+punc, newstest2015, average of 6).

\section{Results}

We submitted 2 Russian-English and 2 EnglishRussian systems for evaluation, each employing a different decoding strategy. Each system is described below. Automatically scored results reported in BLEU (Papineni et al., 2002) for our submission systems can be found in Table 7 for Russian-English and Table 8 for English-Russian.

Finally, as part of WMT16, the results of our submission systems were ranked by monolingual human judges against the machine translation output of other WMT16 participants. These judgments are reported in WMT (2016).

\subsection{Russian-English}

For both Russian-English system submissions, we reused the BigLM15 concept from our WMT15 submissions to build a monolithic language model from the following sources: Yandex ${ }^{4}$, Commoncrawl (Smith et al., 2013), LDC Gigaword English v5 (Parker et al., 2011) and News Commentary. Submission system 1 included the data selected from the large Commoncrawl corpus as outlined in $\S 2.4$, while submission system 2 used this data to build a separate, complementary language model.

For submission system 1, we used a standard phrase based approach with the following parameters/features: distortion-limit of 8 , no reordering over punctuation, hierarchical mslr reordering model (Galley and Manning, 2008), order 7 operational sequence model (Durrani et al., 2011), and a factored language model over the NYT Gigaword corpus with 600 word classes. We incorporated our Tensorflow Neural MT system in via reranking, and applied transliteration as backoff phrase tables during decoding. Lowercased out-

\footnotetext{
${ }^{4}$ https://translate yandex.ru/corpus?lang=en
} 


\begin{tabular}{lcc}
\hline System & Cased BLEU & Unc. BLEU \\
\hline 1. pb + NMT rescore + BigLM(inc. CC data) + Neural translit & 27.6 & 28.8 \\
$\begin{array}{l}\text { 2. pb (clean data) + NMT rescore + CC subsel LM + Neural translit } \\
\quad+\text { Moses translit }\end{array}$ & 27.0 & 28.4 \\
\hline
\end{tabular}

Table 7: Russian-English MT Submission Systems decoding newstest2016

put was recased via neural network. A breakdown of scores for submission system one is indicated in Table 6.

For submission system 2, we used the same approach as system 1, removing the class-factored language model and utilizing both the BigLM used in our WMT15 systems and a secondary language model built from data selected from the monolingual CommonCrawl corpus as outlined in $\$ 2.4$. While this system did use the same transliteration backoff phrase tables to handle OOVs, due to different preprocessing methodologies, some OOVs still remained in the output. The Moses unsupervised statistical transliterator was applied as a postprocess. Finally, the Moses statistical recaser was employed to recase the data before scoring.

\subsection{English-Russian}

Both English-Russian submission systems used a language model interpolated from individual models built from all available Russian data.

Submission system 1 is a standard baseline system employing hierarchical lexicalized reordering and an order 5 operation sequence model.

For submission system 2, we applied factored verb annotation on the training data to guide inflection choice, as outlined in $\S 2.7$. This system also employed hierarchical lexicalized reordering and an order 5 operation sequence model. While this system did not perform as well as system 1, we are interested to see the effect of this verbannotation approach on the human-ranking portion of the evaluation.

Due to time and processing constraints we did not employ Neural Machine Translation rescoring

\begin{tabular}{lcc}
\hline System & Cased BLEU & Unc. BLEU \\
\hline 1. enru-pb & 23.42 & 23.52 \\
2. enru-pb-facvban0 & 20.90 & 21.00 \\
\hline
\end{tabular}

Table 8: English-Russian MT Submission Systems decoding newstest2016 in our English-Russian submission systems.

\section{Conclusion}

We present a series of improvements to our Russian-English and English-Russian machine translation systems. These include general improvements in working with large data sets (language model selection, Drem optimization, neural model rescoring) as well as improvements in language-specific processing (inflection selection/generation, NE transliteration, and neural network recasing). While these innovations show promise in addressing relevant issues in RussianEnglish and English-Russian MT, the overall MT results show that more work is needed to integrate these methods.

\section{Acknowledgements}

We wish to thank the anonymous reviewers for their comments and insight.

\section{References}

Michael Auli, Michel Galley, Chris Quirk, and Geoffrey Zweig. 2013. Joint language and translation modeling with recurrent neural networks. In $E M N L P$, volume 3, page 0 .

Dzmitry Bahdanau, Kyunghyun Cho, and Yoshua Bengio. 2014. Neural machine translation by jointly learning to align and translate. CoRR, abs/1409.0473.

Nikolay Bogoychev and Hieu Hoang. 2016. Fast and highly parallelizable phrase table for statistical machine translation. In Proc. of the First Conference on Statistical Machine Translation (WMT'16), Berlin, Germany, August.

Jacob Devlin, Rabih Zbib, Zhongqiang Huang, Thomas Lamar, Richard Schwartz, and John Makhoul. 2014. Fast and robust neural network joint models for statistical machine translation. In Proc. of the 52nd Annual Meeting of the Association for Computational

Opinions, interpretations, conclusions and recommendations are those of the authors and are not necessarily endorsed by the United States Government. Cleared for public release on 28 Apr 2016. Originator reference number RH-16-115466. Case number 88ABW-2016-2186. 
Linguistics (Volume 1: Long Papers), Proc. of the ACL, Long Papers, pages 1370-1380, Baltimore, MD, USA.

John Duchi, Elad Hazan, and Yoram Singer. 2011. Adaptive subgradient methods for online learning and stochastic optimization. The Journal of Machine Learning Research, 12:2121-2159.

Nadir Durrani, Helmut Schmid, and Alexander Fraser. 2011. A joint sequence translation model with integrated reordering. In Proc. of the 49th Annual Meeting of the Association for Computational Linguistics (ACL '11), pages 1045-1054, Portland, Oregon, June.

Nadir Durrani, Hassan Sajjad, Hieu Hoang, and Philipp Koehn. 2014. Integrating an unsupervised transliteration model into statistical machine translation. In Proc. of the 14th Conference of the European Chapter of the Association for Computational Linguistics, volume 2: Short Papers, pages 148-153, Gothenburg, Sweden, April. Association for Computational Linguistics.

Grant Erdmann and Jeremy Gwinnup. 2015. Drem: The AFRL submission to the WMT15 tuning task. In Proc. of the Tenth Workshop on Statistical Machine Translation, pages 422-427, Lisbon, Portugal, September.

Michel Galley and Christopher D. Manning. 2008. A simple and effective hierarchical phrase reordering model. In Proc. of the Conference on Empirical Methods in Natural Language Processing, EMNLP '08, pages 848-856.

Jeremy Gwinnup, Tim Anderson, Grant Erdmann, Katherine Young, Christina May, Michaeel Kazi, Elizabeth Salesky, and Brian Thompson. 2015. The AFRL-MITLL WMT15 system: There's more than one way to decode it! In Proc. of the Tenth Workshop on Statistical Machine Translation, pages 112-119, Lisbon, Portugal, September.

Marcin Junczys-Dowmunt. 2012. Phrasal rankencoding: Exploiting phrase redundancy and translational relations for phrase table compression. Prague Bulletin of Mathematical Linguistics, 98:6374.

Michaeel Kazi, Brian Thompson, Elizabeth Salesky, Tim Anderson, Grant Erdmann, Eric Hansen, Brian Ore, Katherine Young, Jeremy Gwinnup, Michael Hutt, and Christina May. 2015. The MITLL-AFRL IWSLT-2015 systems. In Proc. of the 11th International Workshop on Spoken Language Translation (IWSLT'15), Da Nang, Vietnam, December.

Katrin Kirchhoff, Yik-Cheung Tam, Colleen Richey, and Wen Wang. 2015. Morphological modeling for machine translation of english-iraqi arabic spoken dialogs. In Proc. of the 2015 Conference of the North American Chapter of the Association for Computational Linguistics: Human Language Technologies, pages 995-1000, Denver, Colorado, May-June.
Dan Klein and Christopher D. Manning. 2003. Accurate unlexicalized parsing. In Proc. of the 41st Annual Meeting of the Association for Computational Linguistics, pages 423-430, Sapporo, Japan, July.

Philipp Koehn, Hieu Hoang, Alexandra Birch, Chris Callison-Burch, Marcello Federico, Nicola Bertoldi, Brooke Cowan, Wade Shen, Christine Moran, Richard Zens, Chris Dyer, Ondřej Bojar, Alexandra Constantin, and Evan Herbst. 2007. Moses: Open source toolkit for statistical machine translation. In Proc. of the 45th Annual Meeting of the ACL on Interactive Poster and Demonstration Sessions, ACL '07, pages 177-180.

Minh-Thang Luong, Hieu Pham, and Christopher D Manning. 2015. Effective approaches to attentionbased neural machine translation. arXiv preprint arXiv:1508.04025.

Kishore Papineni, Salim Roukos, Todd Ward, and WeiJing Zhu. 2002. BLEU: A method for automatic evaluation of machine translation. In Proc. of the 40th Annual Meeting of the Association for Computational Linguistics (ACL '02), pages 311-318, Philadelphia, Pennsylvania, July.

Robert Parker, David Graff, Junbo Kong, Ke Chen, and Kazuaki Maeda. 2011. English Gigaword Fifth Edition LDC2011T07. Philadelphia: Linguistic Data Consortium.

Maja Popović. 2011. Hjerson: An open source tool for automatic error classification of machine translation output. The Prague Bulletin of Mathematical Linguistics (PBML), 96:59-68, 10.

Maja Popović. 2015. chrF: character n-gram F-score for automatic MT evaluation. In Proc. of the Tenth Workshop on Statistical Machine Translation, pages 392-395, Lisbon, Portugal, September.

Jason R. Smith, Herve Saint-Amand, Magdalena Plamada, Philipp Koehn, Chris Callison-Burch, and Adam Lopez. 2013. Dirt cheap web-scale parallel text from the common crawl. In Proc. of the 51st Annual Meeting of the Association for Computational Linguistics (ACL '13), pages 1374-1383, Sofia, Bulgaria, August.

Ilya Sutskever, Oriol Vinyals, and Quoc V Le. 2014. Sequence to sequence learning with neural networks. In Advances in neural information processing systems, pages 3104-3112.

Oriol Vinyals, Łukasz Kaiser, Terry Koo, Slav Petrov, Ilya Sutskever, and Geoffrey Hinton. 2015. Grammar as a foreign language. In Advances in Neural Information Processing Systems, pages 2755-2763.

WMT. 2016. Findings of the 2016 Conference on Statistical Machine Translation. In Proc. of the First Conference on Statistical Machine Translation (WMT '16), Berlin, Germany, August. 